\title{
SPH Model for Numerical Test of Heterogeneous Rock-like Material
}

\author{
Chaoqun Sun ${ }^{1, *}$, Erlong Song ${ }^{2}$, Luchen Zhang ${ }^{1}$ and Feng Nan $^{1}$ \\ ${ }^{1}$ School of Civil Engineering, Shandong Jiaotong University, 250357 Jinan, Shandong Province, China \\ ${ }^{2}$ Road \& Bridge International Co., Ltd., 101100 Tongzhou District, Beijing, China
}

\begin{abstract}
Based on the Smoothed Particles Hydrodynamics (SPH) method, a heterogeneous material numerical model is developed for simulation of fracture process of heterogeneous rock-like material, such as rock. The self-developed SPH program is proposed to analyse the mechanical properties of rock-like materials. In the present program, the combined Mohr-Coulomb criterion is adopted. The deformation of rock-like material under uniaxial compression can be analysed, and the acoustic emission in rock-like material can also be clarified. The calculation results show that the SPH simulation model can be used for numerical test of heterogeneous rock-like material. The process of destruction of rock specimen is evolutionary process. Rock acoustic emission records the brittle failure process of rock-like material.
\end{abstract}

\section{Introduction}

A large number of rock and rock like materials (such as concrete, gypsum, etc.) used in engineering are heterogeneous materials, and there are a large number of tiny defects such as voids, holes, cracks, impurities and so on. When the material is subjected to external force, the internal defects gradually develop into microcracks. With the continuous action of external load, microcracks will continue to expand and eventually break through. Therefore, the failure process of engineering materials is actually the process of continuous accumulation of internal micro defects. The failure process can be divided into three stages: linear elastic deformation stage, plastic deformation stage and post peak deformation stage $^{[1]}$. The traditional numerical method is very effective in the elastic stage, while the numerical simulation method in the plastic deformation and post peak deformation stage is still in constant exploration and research. At the same time, the researchers pay more and more attention to the research through numerical methods. At present, many scholars have carried out research on rock numerical simulation, and used numerical software such as $\mathrm{PFC}^{[2]}, \mathrm{FLAC}^{[3]}, \mathrm{RFPA}^{[4]}$, ANSYS $^{[5]}$, etc., which can achieved good numerical simulation results. However, the continuum method is not suitable for the distortion and crack extension of discontinuous deformation, while the discrete element method can not deal with the continuous deformation effectively. In this paper, SPH (smoothed particles dynamics) method is introduced to simulate the uniaxial compression test of heterogeneous rock like materials, which is a new attempt.

SPH method originated in 1970 s, but it didn't get rapid development until 1990s. At present, SPH method is mostly used in numerical simulation of impact explosion, crack expend and free surface flow. In SPH method, the numerical model is discretized into particles instead of mesh, which does not need to deal with node coupling, elements killing, repartition, etc., which reduces the difficulty of calculation ${ }^{[6-10]}$.

Based on these results, a numerical model of heterogeneous rock like materials is proposed in this paper, and a numerical simulation program is developed based on SPH method to simulate the failure evolution of rock like materials under uniaxial compression test.

\section{Basic principle of SPH method}

\subsection{Kernel approximation function}

The SPH method directly discretizes the solution domain into a series of unconnected points, and constructs an approximate function to solve the partial differential equation. The basic idea is to make every node in the solution domain satisfy the control equation, without the help of grid. The integral expression of the function $f(\mathrm{x})$ that needs to be solved can be written as follows:

$$
f(x) \approx \int_{\Omega} f\left(x^{\prime}\right) W\left(x-x^{\prime}, h\right) \mathrm{d} x^{\prime}
$$

Where, $\boldsymbol{W}$ is called kernel function, and the most commonly used is cubic spline function (B-spline function): 


$$
W(R, h)=\alpha_{d} \times\left\{\begin{array}{cc}
\frac{2}{3}-R^{2}+\frac{1}{2} R^{3}, & 0 \leq R<1 \\
\frac{1}{6}(2-R)^{3}, & 1 \leq R<2 \\
0, & R \geq 2
\end{array}\right.
$$

Where, $\alpha_{d}$ is $h, \frac{15}{7 \pi h^{2}}$ and $\frac{3}{2 \pi h^{3}}$ in $1 \mathrm{D}, 2 \mathrm{D}$ and $3 \mathrm{D}$ respectively. $h$ is the length of influence region of kernel function $\boldsymbol{W}$. At the same time, since $\boldsymbol{W}$ is not a Dirac function, the integral expression of formula (1) can only be approximate, which is the initial form of kernel approximation.

\subsection{Particle equation with material strength}

The total stress tensor $\sigma^{\alpha \beta}$ with material properties is expressed as:

$$
\sigma^{\alpha \beta}=-p \delta^{\alpha \beta}+\tau^{\alpha \beta}
$$

Where $p$ is isotropic pressure and $\tau$ is viscous shear stress.

In solid mechanics, stress can be written as a function of strain and strain rate. Under the assumption of small deformation, the stress rate is proportional to the strain rate, and the ratio coefficient is shear modulus.

$$
\dot{\tau}=G \bar{\varepsilon}^{\alpha \beta}=G\left(\dot{\varepsilon}^{\alpha \beta}-\frac{1}{3} \delta^{\alpha \beta} \dot{\varepsilon}^{\gamma \gamma}\right)
$$

Where, $\bar{\varepsilon}^{\alpha \beta}$ is the shear deformation part of the strain rate; $G$ is the shear modulus; $\dot{\tau}$ is the stress rate; $\dot{\varepsilon}^{\alpha \beta}$ is the strain rate tensor, whose SPH particle form is as follows:

$$
\dot{\varepsilon}^{\alpha \beta}=\frac{1}{2} \sum_{j=1}^{N}\left(\frac{m_{j}}{\rho_{j}} \mathbf{v}_{j i}^{\alpha} \frac{\partial W_{i j}}{\partial \mathbf{x}_{i}^{\beta}}+\frac{m_{j}}{\rho_{j}} \mathbf{v}_{j i}^{\beta} \frac{\partial W_{i j}}{\partial \mathbf{x}_{i}^{\alpha}}\right)
$$

According to the Incremental Plasticity Theory, the strain rate of elastic-plastic materials can be divided into two parts: one is the elastic strain rate $\dot{\varepsilon}_{e}$, the other is the plastic strain rate $\dot{\varepsilon}_{p}$,

$$
\dot{\varepsilon}^{\alpha \beta}=\dot{\varepsilon}_{e}^{\alpha \beta}+\dot{\varepsilon}_{p}^{\alpha \beta}
$$

According to Hooke's law, the elastic strain rate $\dot{\varepsilon}_{e}$ is,

$$
\dot{\varepsilon}_{e}^{\alpha \beta}=\frac{\dot{s}^{\alpha \beta}}{2 G}+\frac{1-2 v}{E} \dot{\sigma}^{m} \delta^{\alpha \beta}
$$

According to the plastic flow rule, the plastic strain part $\dot{\varepsilon}_{p}$ is,

$$
\dot{\varepsilon}_{p}^{\alpha \beta}=\dot{\lambda} \frac{\partial \mathbf{J}}{\partial \sigma^{\alpha \beta}}
$$

Where, $\dot{s}^{\alpha \beta}$ is partial stress tensor; $G$ is shear modulus, $E$ is elastic modulus; $v$ is Poisson's ratio; and $\dot{\sigma}^{m}$ is average stress; $\dot{\lambda}$ is plastic flow factor, which can be determined by the consistency condition; $\boldsymbol{J}$ is the plastic potential function.

According to the above equation, the constitutive relation of particles can be obtained by introducing the Jaumman stress rate to deal with large deformation,

$$
\begin{aligned}
\frac{\mathrm{d} \sigma_{i}^{\alpha \beta}}{\mathrm{d} t} & =\dot{\sigma}_{i}^{\alpha \gamma} \dot{\omega}_{i}^{\beta \gamma}+\dot{\sigma}_{i}^{\gamma \beta} \dot{\omega}_{i}^{\alpha \gamma}+2 G_{i} \dot{e}_{i}^{\alpha \beta}+K_{i} \dot{\varepsilon}_{i}^{\gamma \gamma} \delta_{i}^{\alpha \beta} \\
& -\dot{\lambda}_{i}\left[9 K_{i} \sin \theta_{i} \delta^{\alpha \beta}+\left(G_{i} / \sqrt{J_{2}}\right) \dot{s}_{i}^{\alpha \beta}\right]
\end{aligned}
$$

In the formula, $\dot{e}_{i}^{\alpha \beta}$ is the partial strain rate tensor; $\theta_{i}$ is the shear expansion angle, $\alpha$ and $\beta$ are taken as constant and the value is 1,2 and 3 ; The plastic flow factor $\dot{\lambda}_{i}$ is expressed as follows,

$$
\dot{\lambda}_{i}=\frac{3 \alpha K_{i} \dot{\varepsilon}_{i}^{\gamma \gamma}+\left(G_{i} / \sqrt{J_{2}}\right) \dot{s}_{i}^{\alpha \beta} \dot{\varepsilon}_{i}^{\alpha \beta}}{27 \alpha K_{i} \sin \theta_{i}+G_{i}}
$$

\section{Numerical simulation model}

\subsection{Constitutive relation}

The macroscopic mechanical properties of rock like materials such as rock and concrete are the whole form of mechanical properties of internal micro particles. For a single particle on the mesoscale, its physical and mechanical properties are unique, so it is assumed that all particles in the numerical model have a unified constitutive relationship, only the elastic modulus of particles is different. Therefore, in the numerical model of this paper, the following assumptions are made: (1) the particles of rock like materials are all elastic-plastic media on the meso level; (2) the elastic modulus of particles is a non-uniform parameter and follows the Weibull distribution.

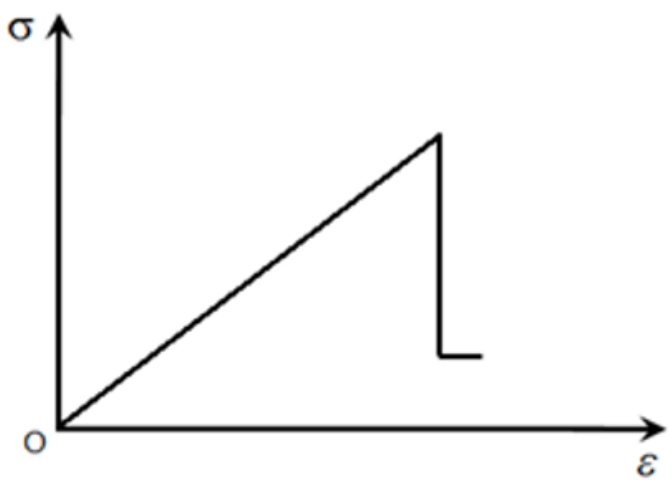

Fig. 1. Constitutive curve of particle model.

\subsection{Constitutive relation}

Based on the basic idea of SPH method, the numerical models are all discrete into unconnected particles. In this paper, the two-dimensional plane strain model is used in the numerical experiment. The size of the model is $50 \mathrm{~mm} \times 100 \mathrm{~mm}$. The model is divided into $80 \times 160$ particles without considering the influence of the boundary effect. The left and right sides of the model are free boundaries, the lower side is fixed, the upper side is applied with a constant loading speed of $0.2 \mathrm{~m} / \mathrm{s}$, the calculation timestep is $1 \times 10-9 \mathrm{~s}$, the expected value of elastic modulus is $E=40 \mathrm{GPa}$, the Poisson's ratio is 0.23 , and the distribution pattern is shown in figure 2 and 3. 


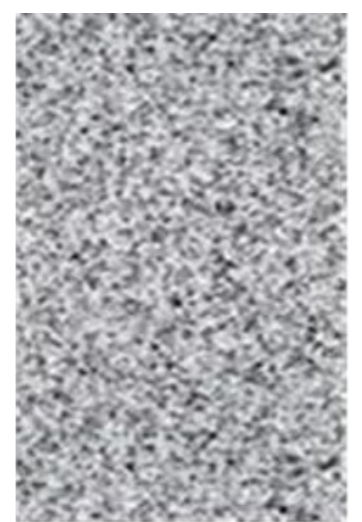

Fig. 2. Experiment model.

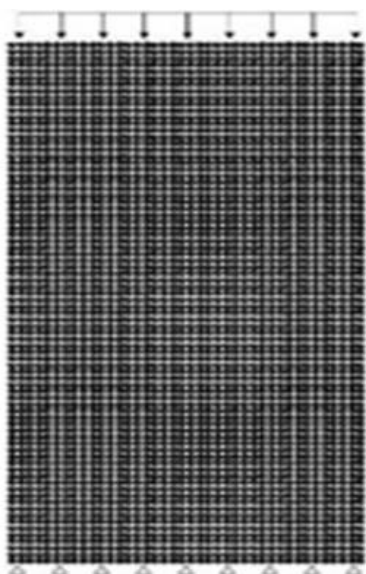

Fig. 3. Numerical model.

\section{Analysis of numerical test results}

In this paper, the heterogeneity numerical model with the degree of homogeneity being 7 is used for calculation. The self-developed SPH program is used to simulate the uniaxial compression test of rock specimen. According to the conclusion of literature [2] and [11], it can be concluded that the specimen whose homogeneity is 7 has obvious brittleness. The acoustic emission statistical program is added to the calculation program in this paper. By recording the axial stress, strain and the AE number of particles with the process of loading, the stress-strain curve and the acoustic emission number in the process of uniaxial loading can be obtained. The numerical results are shown in Figure 4.

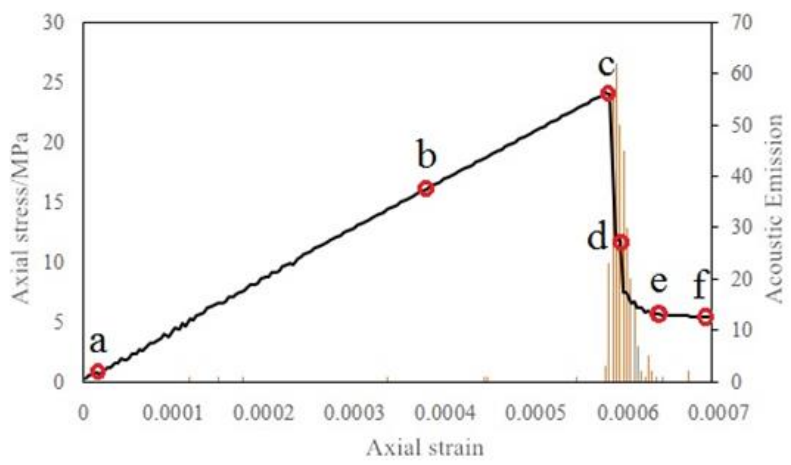

Fig. 4. Stress-strain curve of specimen during loading.
From the changes of stress-strain curve and acoustic emission number, it can be concluded that: (1) in the early stage of loading, the specimen is in the elastic stage, in which the stress does not reach the yield strength of particles. Particles will not be damaged in this stage, and there is no acoustic emission signal. As the loading continues, yield failure happened in a few particles and a small amount of acoustic emission signal appeared; When the axial stress reaches the peak value, the stressstrain curve falls sharply, and a large number of acoustic emission signals are generated, which indicated that abundant particles in the test specimen are damaged in a very short time and the acoustic emission signals are released. The drop of the curve shows that the bearing capacity of the test specimen is sharply reduced, indicating that the rock specimen has brittle failure properties. After that, the specimen loses the ability of bearing.

In the stress-strain curve, take the acoustic emission recording diagram of the specimen corresponding to points $\mathrm{a}, \mathrm{b}, \mathrm{c}, \mathrm{d}$, e and $\mathrm{f}$, as shown in Figure 5 below:

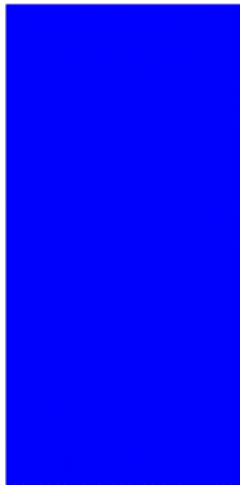

a

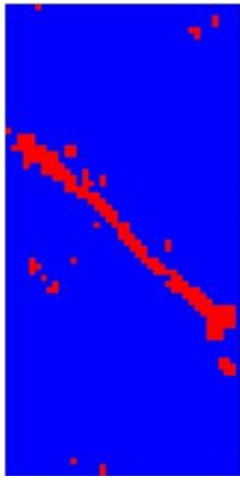

d

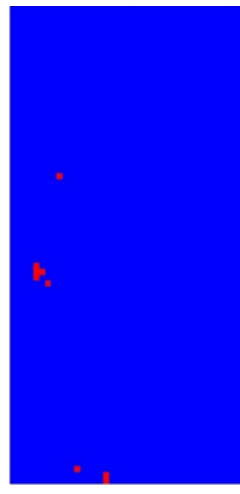

b

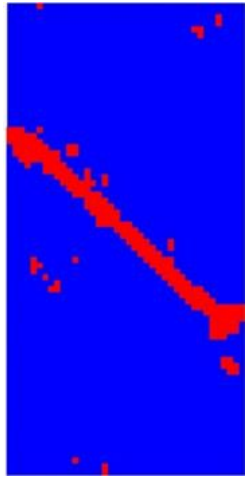

e
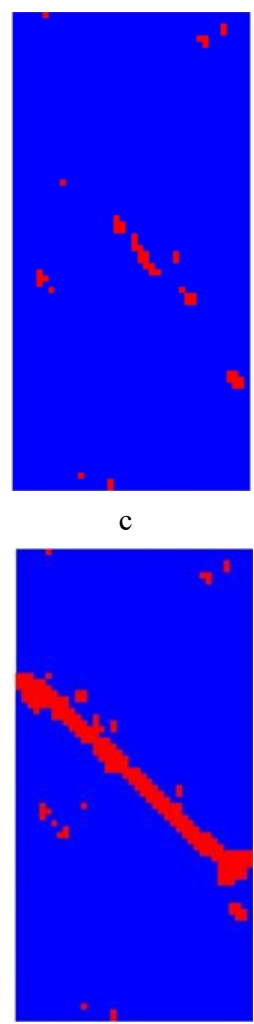

f
Fig. 5. Acoustic emission nephogram of loading process.

Corresponding to the position of each point in the stress-strain curve, point a is at the initial loading stage of specimen, without particle damage and acoustic emission signal appear; at point $b$, a small number of particles in the specimen generate acoustic emission signal; when the axial stress reaches the peak value of point $\mathrm{c}$, the number of yield particles in the specimen slightly increases; at point $d$, a large number of damage particle occurred; at point e, the brittle failure of the specimen has been formed; and the change of point $f$ relative to point e is not obvious. From point a to point e, the process of specimen's brittle failure can be clearly 
demonstrated. The meso-scale failure of particles finally forms the macro fracture surface which can also be obtained by numerical simulation. The calculation results in this paper are consistent with those in [2] and [11].

\section{Conclusion}

According to the calculation theory of SPH method, this paper presents a numerical calculation method which can be used to simulate the heterogeneous rock like materials such as rock and concrete. The numerical simulation of the uniaxial compression test of the heterogeneous rock like materials is carried out by using the self-developed calculation program. The conclusions are as follows:

(1) The numerical model based on SPH method can be used in the numerical simulation of rock, concrete, coal and other materials;

(2) The simulation results can get the meso-scale damage and macro damage state of heterogeneous rock like materials under load, and reveal the stress evolution process of rock like materials at the meso-scale level.

\section{Acknowledgment}

Authors wish to thank the financial support from Joint project of Natural Science Foundation of Shandong Province (ZR2017LEE025, ZR2017LEE029) and Shandong Jiaotong University 'Climbing' Research Innovation Team Program (SDJTUC1801), so that this research can be carried out well.

\section{References}

1. Cai M., Kaiser P., Morioka H., et al., INT J ROCK MECH MIN, 44, 550-564, (2007)

2. Zhou Y, Wu S., Xu X, et al., Chinese Journal of Rock Mechanics and Engineering 32(5): 951959,( 2013)

3. Chai J., Gao Y., Wu S., et al, Journal of Mining \& Safety Engineering, 33, 528-534, (2016)

4. Chong Z., Li X., Hou P., et al., International Journal of Mining Science and Technology, 27, 507-515, (2017).

5. Caplar R and Kulisic P Proc. Int. Conf. on Nuclear Physics (Munich) vol 1 p 517, (1973)

6. Guilkey J.E., Harman T. B., Banerjee B.,2007, Computers \& Structures, 85(11-14), 660-674.

7. Batra R.C., Zhang G.M., Computational Mechanics, 40(3), 531-546, (2007).

8. Roubtsova V, Kahawita R, Computers \& Fluids, 35(10), 1359-1371, (2006)

9. G.R.Liu, M.B.Liu., World Scientific Publishing Company, (2003).

10. HAN T., ZHANG J.., Chinese Journal of Rock Mechanics and Engineering, 33(S1), 31983204,(2014).

11. Sun C., Cheng G., Li S., et al., Journal of China Coal Society, 39(11), 2183-2189, (2014). 